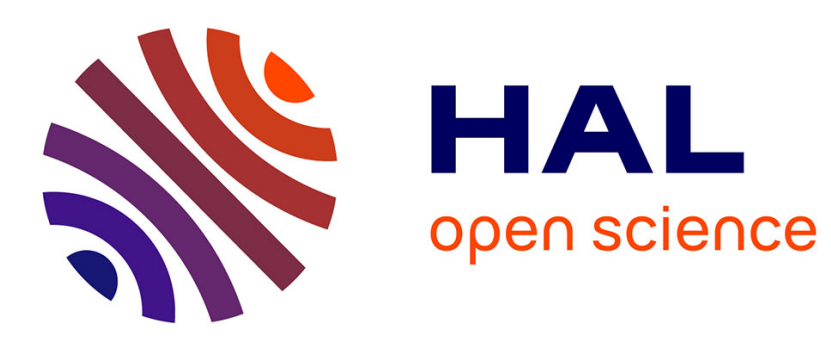

\title{
La microanalyse de l'azote par l'observation directe de réactions nucléaires applications
}

\author{
G. Amsel, D. David
}

\section{To cite this version:}

G. Amsel, D. David. La microanalyse de l'azote par l'observation directe de réactions nucléaires applications. Revue de Physique Appliquée, 1969, 4 (3), pp.383-391. 10.1051/rphysap:0196900403038300 . jpa-00243300

\section{HAL Id: jpa-00243300 https://hal.science/jpa-00243300}

Submitted on 1 Jan 1969

HAL is a multi-disciplinary open access archive for the deposit and dissemination of scientific research documents, whether they are published or not. The documents may come from teaching and research institutions in France or abroad, or from public or private research centers.
L'archive ouverte pluridisciplinaire HAL, est destinée au dépôt et à la diffusion de documents scientifiques de niveau recherche, publiés ou non, émanant des établissements d'enseignement et de recherche français ou étrangers, des laboratoires publics ou privés. 


\title{
LA MIGROANALYSE DE L'AZOTE PAR L'OBSERVATION DIREGTE DE RÉAGTIONS NUGLÉAIRES APPLIGATIONS ( $\left.{ }^{1}\right)$
}

\author{
Par G. AMSEL et D. DAVID, \\ Groupe de Physique des Solides de l'École Normale Supérieure $\left({ }^{2}\right)$, Tour 23, 9, quai Saint-Bernard, Paris, $5^{\mathrm{e}}$.
}

(Reçu le 24 mars 1969.)

\begin{abstract}
Résumé. - La méthode de microanalyse par l'observation directe de réactions nucléaires a été appliquée au dosage de l'azote 14 . Le spectre caractéristique de cet isotope, bombardé par des deutons, a été étudié à diverses énergies. Les sections efficaces différentielles des réactions observées, de type $(\mathrm{d}, \mathrm{p})$ et $(\mathrm{d}, \alpha)$, ont été mesurées entre 600 et $1400 \mathrm{keV}$. Le pic de la réaction $\mathrm{N}^{14}(\mathrm{~d}, \alpha) \mathrm{C}^{12}$, dont l'énergie est d'environ $10 \mathrm{MeV}$, est très isolé et permet de doser l'azote en présence de la plupart des autres éléments. D'autres pics du spectre peuvent être utilisés en l'absence de noyaux tels que $\mathrm{B}^{10}, \mathrm{~F}^{19}$, et fournissent des taux de comptage supérieurs. La sensibilité de cette méthode d'analyse non destructive est de l'ordre de $10^{-3} \mu \mathrm{g} / \mathrm{cm}^{2}$. Un échantillon d'une surface de $1 \mathrm{~mm}^{2}$ peut être dosé aisément. Les mesures quantitatives sont rendues absolues par l'utilisation d'étalons de nitrure de tantale. L'application de ces techniques à l'étude de la pulvérisation cathodique réactive permet de mettre en évidence l'influence de la pression partielle d'azote sur la composition des dépôts de nitrures. D'autres applications à l'étude de la nitruration à chaud de divers métaux sont présentées.
\end{abstract}

\begin{abstract}
Microanalysis by the direct observation of nuclear reactions has been applied to the determination of nitrogen 14 . The energy spectrum corresponding to this isotope, when bombarded with deuterons, was studied at various energies. The differential crosssections of the $(d, p)$ and $(d, \alpha)$ type reactions induced were measured from $600 \mathrm{keV}$ to $1400 \mathrm{keV}$. The peak from the reaction $\mathrm{N}^{14}(\mathrm{~d}, \alpha) \mathrm{C}^{12}$, at an energy of about $10 \mathrm{MeV}$, is well isolated and allows the determination of nitrogen in the presence of most of the other nuclei. Other peaks of the spectrum may be used when isotopes like $\mathrm{B}^{10}$ or $\mathrm{F}^{19}$ are not present in the sample ; counting rates may then be higher. Sensitivity of this non destructive analytical method is of the order of $10^{-3} \mu \mathrm{g} / \mathrm{cm}^{2}$. A $1 \mathrm{~mm}^{2}$ sample may be easily analyzed. Using tantalum nitride as a standard, an absolute measurement of the amount of nitrogen is obtained. The application of these techniques to the study of reactive sputtering allowed us to show the influence of nitrogen partial pressure on the composition of the nitride films formed. Other applications to the study of the nitration of various metals in hot ammonia are also described.
\end{abstract}

Introduction. - La méthode de microanalyse des éléments légers par l'observation directe de réactions nucléaires, mise au point et utilisée de façon routinière pour les isotopes 16 et 18 de l'oxygène [1-5], s'applique également aux isotopes de l'azote. Les mérites de cette méthode comparée à d'autres, telles que l'analyse par activation ou les sondes électronique ou ionique, ont été discutés dans les références [2] et [5]. Rappelons brièvement que cette technique consiste à observer les particules chargées (protons, rayons $\alpha$ ), émises lors du bombardement d'un échantillon par un faisceau

(1) Travail effectué avec le soutien du C.N.R.S. (RCP no 157), de la D.R.M.E. et de la D.G.R.S.T.

(2) Laboratoire associé au Centre National de la Recherche Scientifique. de particules chargées (protons, deutons, etc.). La détection de ces particules est effectuée au moyen de détecteurs à semiconducteur ; l'analyse de leur spectre en énergie fournit des pics caractéristiques des éléments légers contenus dans les premiers microns de la cible. La sensibilité est très élevée; la méthode est non destructive et donne, par comparaison à une cible étalon, un dosage global quantitatif très précis, de façon indépendante de la structure de la matrice contenant l'isotope à doser. L'analyse de la forme des pics permet d'atteindre le profil de concentration en fonction de la profondeur de l'isotope étudié, du moins dans les premiers microns de l'échantillon [3-5].

L'abondance naturelle de $\mathrm{N}^{14}$ étant de 99,635\%, le dosage de $\mathrm{N}^{14}$ équivaut à celui de l'élément azote, qui fait l'objet de cet article. Seuls les détails particuliers 
relatifs au dosage de l'azote 14 seront exposés ici; on trouvera dans les références [2] à [5] les détails expérimentaux relatifs à la méthode en général.

Les domaines d'application seront encore nettement plus nombreux quand aura été développé le dosage de l'azote 15. Celui-ci se fera dans des conditions analogues à celles qui sont habituelles pour $\mathrm{O}^{18}[2,3,5]$ et permettra d'effectuer des études par traçage au cours d'expériences réalisées avec des composés azotés enrichis en $\mathrm{N}^{15}$.

I. Réactions utilisées. - Les réactions nucléaires utilisées doivent satisfaire à quelques conditions essentielles : ne nécessiter qu'un faisceau de particules de basse énergie (moins de $2 \mathrm{MeV}$ par unité de charge, donc un petit accélérateur); fournir des particules très énergiques dont la détection est aisée; avoir une valeur $Q$ plus élevée que celle de la plupart des autres réactions analogues sur des noyaux légers, pour éliminer l'effet de réactions parasites sur d'autres noyaux que $\mathrm{N}^{\mathbf{1 4}}$ contenus dans la cible; avoir une section efficace élevée. La réaction $\mathrm{N}^{14}(\mathrm{p}, \alpha) \mathrm{C}^{11}$ ayant une valeur $Q$ négative, notre choix s'est porté sur les réactions $\mathrm{N}^{14}(\mathrm{~d}, \alpha) \mathrm{C}^{12}$ et $\mathrm{N}^{14}(\mathrm{~d}, \mathrm{p}) \mathrm{N}^{15}$, dont les caractéristiques sont données dans le tableau I. Nous allons voir que ces réactions satisfont, dans une large mesure, aux exigences énoncées.

\section{TABLEAU I}

\begin{tabular}{|c|c|c|}
\hline RÉAGTION & $\begin{array}{c}\text { ÉTAT } \\
\text { DU NOYAU } \\
\text { RÉSIDUEL } \\
-\end{array}$ & $\begin{array}{c}Q \\
\left.\text { (en } \begin{array}{c}\mathrm{MeV} \\
-\end{array}\right)\end{array}$ \\
\hline \multirow[t]{5}{*}{$\mathrm{N}^{14}(\mathrm{~d}, \mathrm{p}) \mathrm{N}^{15}$} & $\begin{array}{l}\text { fondamental } \\
\text { 1er excité }^{\text {er }}\end{array}$ & $\begin{array}{l}8,615 \\
3,335\end{array}$ \\
\hline & $2^{\mathrm{e}} \quad »$ & 3,305 \\
\hline & $3^{\mathrm{e}}$ & 2,285 \\
\hline & $4^{e}$ & 1,455 \\
\hline & $5^{\mathrm{e}} \quad »$ & 1,305 \\
\hline \multirow{3}{*}{$\mathrm{N}^{14}(\mathrm{~d}, \alpha) \mathrm{C}^{12}$} & fondamental & 13,579 \\
\hline & $1^{\mathrm{er}}$ excité & 9,146 \\
\hline & $2^{\mathrm{e}} \quad \gg$ & 5,923 \\
\hline
\end{tabular}

A. Description D'un SPeGtre D'Amplitude typique. - La figure 1 représente un spectre d'amplitude typique obtenu en bombardant avec des deutons de $1,2 \mathrm{MeV}$ une cible de nitrure de tantale d'une épaisseur de $1400 \AA$ déposée sur du tantale pur. L'angle d'observation par rapport au faisceau était de $150^{\circ}$; le détecteur était placé à $12 \mathrm{~cm}$ et avait une surface de $3 \mathrm{~cm}^{2}$ avec une zone sensible d'une épaisseur de 300 microns. Un film de mylar de 19 microns placé devant le détecteur absorbait les particules rétrodiffusées. On distingue sur ce spectre les divers pics correspondant aux réactions du tableau I. Ces pics seront désignés respectivement par $p_{0}, p_{1}$, etc., et $\alpha_{0}, \alpha_{1}$, etc.; la liste de ces pics est donnée dans le

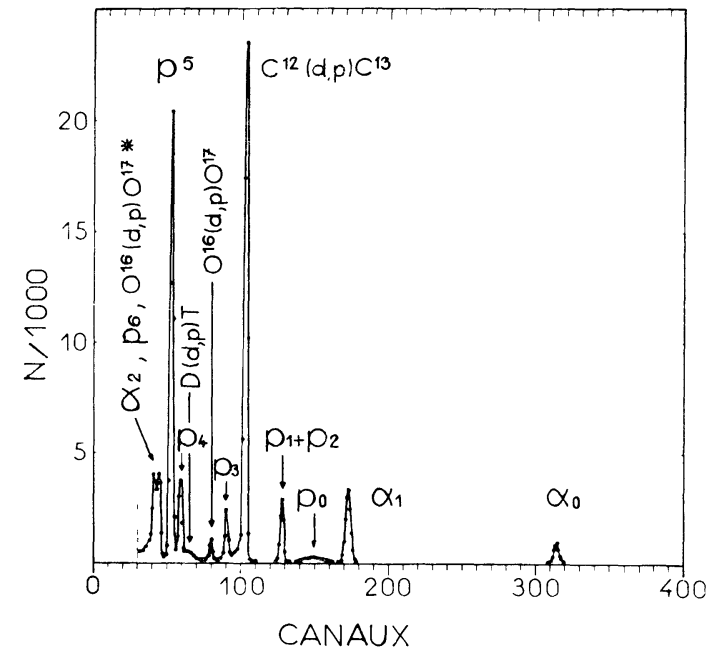

FIG. 1. - Spectre correspondant à une cible de nitrure de tantale de $1400 \AA$, bombardée par des deutons de $1200 \mathrm{keV}$. Observation à $150^{\circ}$, absorbant en mylar de $19 \mu$, zone sensible du détecteur épaisse de $300 \mu \mathrm{m}$, $26 \mathrm{keV}$ par canal.

tableau II, les énergies avant le mylar étant calculées au moyen des relations cinématiques classiques.

Vers le canal 40 , se trouve un pic multiple qui est la combinaison des pics de trois réactions (tableau II) conduisant à des états excités des noyaux résiduels : $\alpha_{2}, P_{6}$ et $\mathrm{O}^{16}(\mathrm{~d}, \mathrm{p}) \mathrm{O}^{17^{*}}$. En effet, comme la résolution en énergie du système à mi-hauteur du pic est d'environ $50 \mathrm{keV}$ pour les protons et $80 \mathrm{keV}$ pour les rayons $\alpha$, ces pics, distants d'environ $50 \mathrm{keV}$, ne peuvent pas être séparés. L'oxygène 16 est présent à titre de contaminant, mais son effet ne peut être négligé, vu la section efficace élevée de la réaction correspondante. Ainsi, ce pic multiple ne peut guère servir au dosage de l'azote 14. Remarquons qu'inversement, lorsqu'on dose $\mathrm{O}^{16}$, il faut faire appel à un autre pic dans les échantillons contenant de l'azote [2].

Le pic suivant $p_{5}$ est le plus intense du spectre de l'azote. Il peut être bien séparé du pic de l'oxygène mais moins bien de $p_{4}$, assez proche. On peut utiliser la somme de ces deux pics pour un dosage à très haut taux de comptage de l'azote. Néanmoins, la sensibilité sera limitée pour de très faibles quantités d'azote. En effet, le pic élargi provenant de la réaction $\mathrm{D}(\mathrm{d}, \mathrm{p}) \mathrm{T}$ fournit un bruit de fond dans cette région, pour des énergies de bombardement supérieures à $1 \mathrm{MeV}$. Le deutérium, apporté par le faisceau de deutons, est toujours présent. Qui plus est, les réactions parasites sont fort nombreuses dans ce domaine d'énergie, comme on le verra. Enfin, la séparation de ce pic n'est possible que pour des cibles suffisamment minces, dans lesquelles l'azote est dans les premiers milliers d'angströms du substrat.

Le pic $p_{3}$, qui suit celui de la réaction $\mathrm{O}^{16}(\mathrm{~d}, \mathrm{p}) \mathrm{O}^{17}$ et en est bien séparé, est peu intense et son utilisation 
TABLEAU II

\begin{tabular}{|c|c|c|c|}
\hline RÉAGTION & Pia & $\begin{array}{c}\text { ÉNERGIE } \\
\text { AVANT MYLAR } \\
(\mathrm{MeV})\end{array}$ & $\begin{array}{c}\text { ÉNERGIE } \\
\text { APRÈs MYLAR } \\
(\mathrm{MeV})\end{array}$ \\
\hline - & - & - & - \\
\hline $\mathrm{N}^{14}(\mathrm{~d}, \alpha) \mathrm{C}^{12}$ & $\alpha_{2}$ & 4,482 & 1,16 \\
\hline $\mathrm{N}^{14}(\mathrm{~d}, \mathrm{p}) \mathrm{N}^{15}$ & $P_{6}$ & 1,734 & 1,20 \\
\hline $\mathrm{O}^{16}(\mathrm{~d}, \mathrm{p}) \mathrm{O}^{17}$ & 1er excité & 1,790 & 1,26 \\
\hline $\mathrm{N}^{14}(\mathrm{~d}, \mathrm{p}) \mathrm{N}^{15}$ & $P_{5}$ & 1,963 & 1,48 \\
\hline$»$ & $P_{4}$ & 2,096 & 1,64 \\
\hline $\mathrm{D}(\mathrm{d}, \mathrm{p}) \mathrm{T}$ & fondamental & 2,306 & 1,88 \\
\hline $\mathrm{O}^{16}(\mathrm{~d}, \mathrm{p}) \mathrm{O}^{17}$ & fondamental & 2,573 & 2,18 \\
\hline $\mathrm{N}^{14}(\mathrm{~d}, \mathrm{p}) \mathrm{N}^{15}$ & $P_{3}$ & 2,835 & 2,48 \\
\hline $\mathrm{C}^{12}(\mathrm{~d}, \mathrm{p}) \mathrm{C}^{13}$ & fondamental & 3,129 & 2,80 \\
\hline $\mathrm{N}^{14}(\mathrm{~d}, \mathrm{p}) \mathrm{N}^{15}$ & $P_{2}$ & 3,746 & 3,47 \\
\hline$»$ & $P_{1}$ & 3,776 & 3,50 \\
\hline $\mathrm{N}^{14}(\mathrm{~d}, \alpha) \mathrm{C}^{12}$ & $\alpha_{1}$ & 6,739 & 4,60 \\
\hline$»$ & $\alpha_{0}$ & 9,880 & 8,38 \\
\hline $\mathrm{N}^{14}(\mathrm{~d}, \mathrm{p}) \mathrm{N}^{15}$ & $P_{0}$ & 8,561 & $8,41(*)$ \\
\hline
\end{tabular}

(*) Rejeté bien plus bas lorsqu'un détecteur mince est utilisé.

offre un intérêt réduit. L'intensité du pic suivant, provenant de la contamination au carbone de la cible, est très variable d'un échantillon à l'autre.

Le pic suivant est la somme de $p_{1}$ et $p_{2}$ qui forment un doublet (écart $30 \mathrm{keV}$ ). Ce pic peu intense ne présente pas d'avantages particuliers. Le large pic qui suit est dû aux protons de $8,4 \mathrm{MeV}$ qui ne sont pas arrêtés dans la zone sensible de 300 microns du détecteur. L'emplacement de ce pic peut être changé à volonté en agissant sur l'épaisseur de cette barrière par la polarisation appliquée au détecteur [6,7]. C'est ainsi que, en ramenant l'épaisseur de la zone sensible à 230 microns, on obtient un spectre ( fig. 2) où le pic dû à $p_{0}$ est rejeté à la gauche du pic $p_{1}+p_{2}$.

Le pic $\alpha_{1}$ qui suit convient particulièrement bien au dosage de l'azote. Il est séparé de $p_{1}$ par plus de $1 \mathrm{MeV}$. Son intensité, tout en étant inférieure à celle de $p_{5}$, est encore bien appréciable. L'absence de bruit de fond dans son voisinage, pour un réglage similaire à celui de la figure 2 , assure une haute sensibilité.

Le pic $\alpha_{0}$ est tout à fait remarquable. Il est séparé de $\alpha_{1}$ par près de $4 \mathrm{MeV}$. Il n'existe pratiquement aucune réaction fournissant un rayon $\alpha$ aussi énergique, ce qui le met à l'abri des réactions parasites. Le pic $p_{0}$ pourrait venir brouiller $\alpha_{0}$ (tableau II) si les protons correspondants étaient arrêtés dans la zone sensible du détecteur. Le choix d'un détecteur à barrière assez mince s'impose donc. Dans les analyses difficiles, ce pic permet toujours un dosage sans aucun bruit de fond. Vu l'intensité de $\alpha_{0}$, plus faible que celle de $\alpha_{1}$, ce dernier est utilisé dans les cas courants, comme nous le montrerons plus loin.

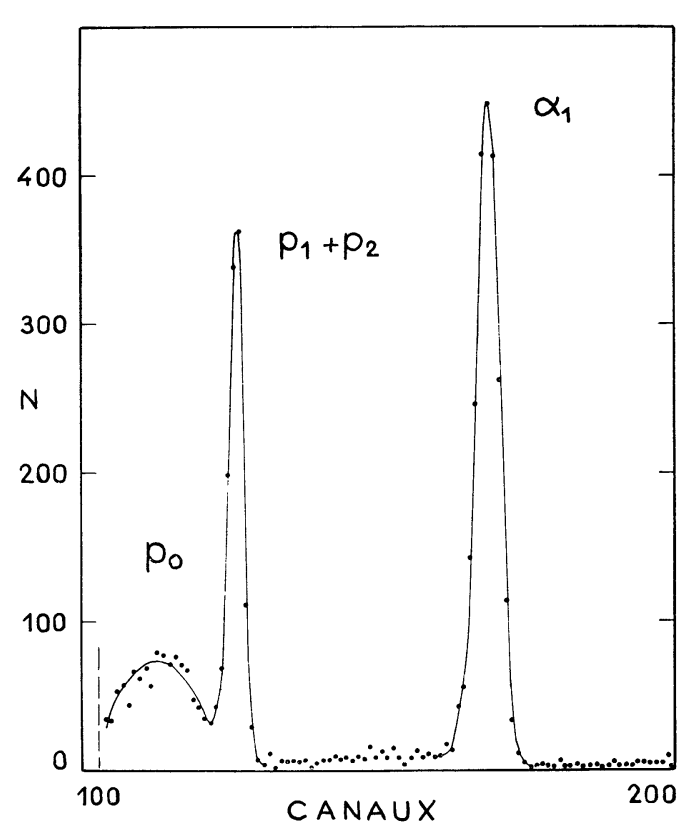

FIG. 2. - Détail d'un spectre analogue à celui de la figure 1 , la zone sensible du détecteur étant ici réduite à $230 \mu$; le pic dégradé $p_{0}$ est passé à la gauche de $p_{1}+p_{2}$.

B. RÉACtions parasites. - Les tableaux III a et III b présentent les valeurs $Q$, par ordre décroissant, des réactions induites par les deutons aux basses énergies sur les noyaux légers. Par simple inspection, on 
TABLEAU III a

\begin{tabular}{|c|c|c|c|}
\hline \multicolumn{4}{|c|}{ RÉAGTIONS $(\mathrm{d}, \alpha)$} \\
\hline ÉLÉMENT & $Q_{0}(\underline{\mathrm{MeV}})$ & $\begin{array}{c}\text { ÉLÉMENT } \\
-\end{array}$ & $Q_{0}(\mathrm{MeV})$ \\
\hline $\mathrm{B}^{10}$ & 17,819 & $\mathrm{Al}^{27}$ & 6,701 \\
\hline $\mathrm{Li}^{7}$ & 14,163 & $\mathrm{Si}^{29}$ & 6,012 \\
\hline $\mathrm{N}^{14}\left(\alpha_{0}\right)$ & 13,579 & $\mathrm{C}^{13}$ & 5,167 \\
\hline $\mathrm{F}^{19}$ & 10,038 & $\mathrm{~S}^{32}$ & 4,890 \\
\hline $\mathrm{O}^{17}$ & 9,812 & $\mathrm{O}^{18}$ & 4,237 \\
\hline $\mathrm{N}^{14}\left(\alpha_{1}\right)$ & 9,146 & $\mathrm{Si}^{30}$ & 3,121 \\
\hline $\mathrm{P}^{31}$ & 8,170 & $\mathrm{O}^{16}$ & 3,116 \\
\hline $\mathrm{B}^{11}$ & 8,022 & $\mathrm{Mg}^{26}$ & 2,909 \\
\hline $\mathrm{N}^{15}$ & 7,683 & $\mathrm{Mg}^{24}$ & 1,964 \\
\hline $\mathrm{Be}^{9}$ & 7,152 & $\mathrm{Si}^{28}$ & 1,421 \\
\hline $\mathrm{Mg}^{25}$ & 7,047 & $\mathrm{C}^{12}$ & $<0$ \\
\hline $\mathrm{Na}^{23}$ & 6,909 & & \\
\hline
\end{tabular}

TABLEAU III b

\begin{tabular}{|c|c|c|c|}
\hline \multicolumn{4}{|c|}{ RÉactions $(\mathrm{d}, \mathrm{p})$} \\
\hline ÉLÉMENT & $Q_{0}(\underline{\mathrm{MeV}})$ & ÉLÉMENT & $Q_{0}(\mathrm{MeV})$ \\
\hline $\mathrm{B}^{10}$ & 9,237 & $\mathrm{Na}^{23}$ & 4,734 \\
\hline $\mathrm{Mg}^{25}$ & 8,873 & $\mathrm{Be}^{9}$ & 4,585 \\
\hline $\mathrm{N}^{14}\left(\mathrm{p}_{0}\right)$ & 8,615 & $F^{19}$ & 4,379 \\
\hline $\mathrm{Si}^{29}$ & 8,390 & $\mathrm{Si}^{30}$ & 4,367 \\
\hline $\mathrm{S}^{32}$ & 6,418 & $\mathrm{Mg}^{26}$ & 4,212 \\
\hline $\mathrm{Si}^{28}$ & 6,253 & $\mathrm{C}^{12}$ & 2,719 \\
\hline $\mathrm{C}^{13}$ & 5,947 & $\mathrm{O}^{16}$ & 1,919 \\
\hline $\mathrm{P}^{31}$ & 5,712 & $\mathrm{O}^{18}$ & 1,731 \\
\hline $\mathrm{O}^{17}$ & 5,842 & $\mathrm{~N}^{14}\left(\mathrm{p}_{5}\right)$ & 1,305 \\
\hline $\mathrm{Al}^{27}$ & 5,499 & $\mathrm{~B}^{11}$ & 1,138 \\
\hline $\mathrm{Mg}^{24}$ & 5,106 & $\mathrm{~N}^{15}$ & 0,267 \\
\hline $\mathrm{Li}^{6}$ & 5,027 & $\mathrm{Li}^{7}$ & $-0,192$ \\
\hline
\end{tabular}

détermine quels sont les éléments gênants lors d'un dosage. Ainsi, pour les réactions $(\mathrm{d}, \alpha)$, il n'y a que l'isotope $\mathrm{B}^{10}$ qui puisse fournir un bruit de fond pour $\alpha_{0}$; en effet, vu sa masse faible, l'isotope $\mathrm{Li}^{7}$, tout en présentant une valeur $Q$ plus élevée que $\alpha_{0}$, ne fournit qu'un rayon $\alpha$ d'énergie voisine de celle de $\alpha_{1}$. Ce dernier pic peut donc être brouillé par $\mathrm{B}^{10}$ et, en plus, par $\mathrm{Li}^{7}$ et $\mathrm{F}^{19} . \mathrm{O}^{17}$ étant très rare (abondance $0,037 \%$ ), il ne peut gêner $\alpha_{1}$ que pour les cibles très riches en oxygène et très pauvres en azote. Cependant, comme nous le verrons, le pic $\alpha_{1}$ peut être perturbé dans certains cas par des phénomènes d'empilement provenant de l'électronique. Pour le dosage avec la réaction (d, p), la situation est beaucoup moins favorable. Le pic $p_{0}$ ne présentant guère d'avantages par rapport à $\alpha_{0}$ ou $\alpha_{1}$, la comparaison des valeurs $Q$ doit être faite avec l'énergie dégagée par $p_{5}$. Les réactions parasites sont ici fort nombreuses; cela ne signifie pas que le dosage est impossible en présence des isotopes correspondants car les pics peuvent être séparés dans les cas favorables.
Aucune règle générale ne peut être donnée, si ce n'est que $p_{5}$ doit être utilisé avec précaution; lorsque le bruit de fond parasite est absent, son utilisation est cependant avantageuse vu sa grande intensité.

II. Cibles de référence. - Les cibles utilisées étaient de deux sortes : cibles minces pour la mesure précise de la variation des sections efficaces en fonction de l'énergie, et cibles d'épaisseur moyenne, mais de teneur en azote bien connue, servant d'étalon pour les dosages.

Les cibles étaient obtenues sur des supports de tantale par pulvérisation cathodique réactive de tantale sous atmosphère d'argon contenant de l'azote. Ce procédé de production de couches de nitrures de tantale, d'usage routinier dans l'industrie des composants électroniques, a fait l'objet d'une étude poussée par les méthodes décrites ici. Les détails relatifs à la construction de ces cibles seront donc exposés dans le chapitre consacré aux applications. L'erreur sur la teneur en azote de nos cibles étalons est, dans l'état d'avancement actuel des travaux, de $\pm 10 \%$. Cette imprécision ne retentit pas sur les mesures relatives, dont la précision ne dépend que de la statistique de comptage et atteint facilement $1 \%[2,5]$.

III. Etude des sections efficaces. - Le choix de l'énergie de bombardement, pour une réaction nucléaire donnée, est en grande partie déterminé par la forme de sa courbe de section efficace et la nature de la mesure à effectuer. Ainsi un dosage global, intéressant toute l'épaisseur d'une couche contenant de l'azote, fait choisir une énergie correspondant à un plateau de la courbe [2]; le rendement de la réaction ne varie alors pas avec la profondeur. Au contraire, opérer dans une région où la section efficace croît rapidement permet de concentrer l'attention sur une mince couche de matière, à la surface de la cible $[3,5]$, en donnant à sa contribution au spectre une part prépondérante.

Nous avons mesuré la section efficace des réactions mentionnées dans le tableau I de 600 à $1400 \mathrm{keV}$, à l'exception de l'état fondamental de $\mathrm{N}^{14}(\mathrm{~d}, \mathrm{p}) \mathrm{N}^{15}$. Les premier et deuxième états excités de cette réaction n'ont pu être séparés. Le troisième état excité, aux énergies inférieures à $800 \mathrm{keV}$, est perturbé par le pic de la réaction $\mathrm{D}(\mathrm{d}, \mathrm{p}) \mathrm{T}$, dont l'emplacement par rapport aux pics de $\mathrm{N}^{14}$ varie avec l'énergie. Il n'a donc pu être étudié qu'à des énergies plus élevées.

A. Conditions expérimentales. - La cible utilisée avait une épaisseur de $1400 \AA$, provoquant une perte moyenne d'énergie d'environ $15 \mathrm{keV}$ pour des deutons de $1 \mathrm{MeV}$. Le point d'impact du faisceau était fréquemment changé pour éviter les altérations ; l'uniformité de la cible a été vérifiée. Le passage en valeur absolue était obtenu par normalisation avec une cible étalon. Compte tenu de toutes les erreurs, la précision sur la valeur absolue de ces sections efficaces est estimée à $\pm 15 \%$. Le détecteur et la géométrie étaient les 
mêmes que pour le spectre de la figure 1. La polarisation du détecteur était modifiée de façon à éloigner le pic dégradé $p_{0}$ des pics observés. Le bombardement de la cible par les deutons donne également lieu à des réactions nucléaires telles que $\mathrm{C}^{12}(\mathrm{~d}, \mathrm{n}) \mathrm{N}^{13}, \mathrm{~N}^{14}(\mathrm{~d}, \mathrm{n}) \mathrm{O}^{15}$, dont les noyaux résiduels sont radioactifs. Ceux-ci émettent donc des électrons, générateurs de nombreuses impulsions parasites de faible amplitude dans la chaîne de mesure. Ces impulsions étaient éliminées au moyen d'un amplificateur à seuil.

B. RÉsultats. - Les sections efficaces différentielles à $150^{\circ}$ dans le système de repère du laboratoire $\left(\sigma\left(150^{\circ}\right)_{\mathrm{lab}}\right)$ sont représentées sur les figures 3,4 et 5 . On constate ( fig. 3 ) que le pic $p_{5}$ reste le plus intense

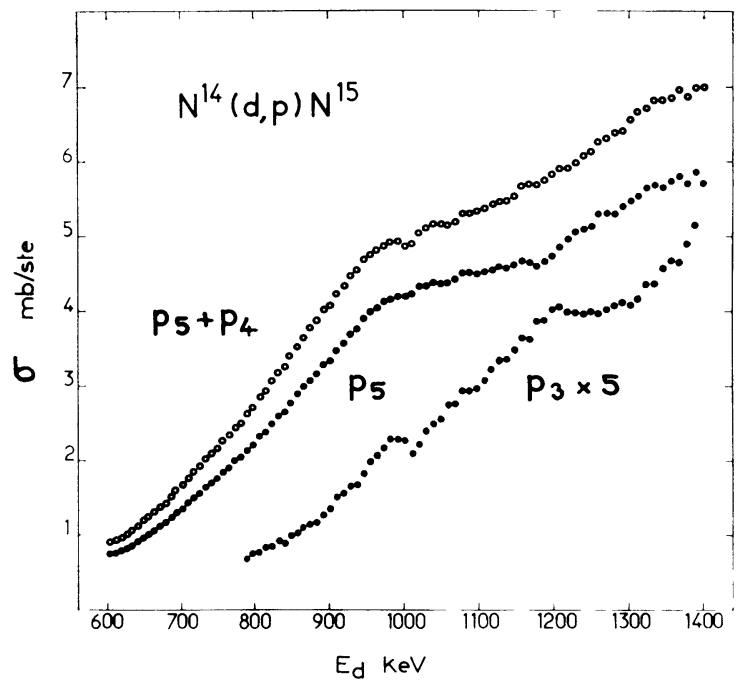

FIG. 3. - Sections efficaces différentielles à $150^{\circ}$ des pics $p_{3}, p_{5}$, et de l'ensemble $\left(p_{5}+p_{4}\right)$.

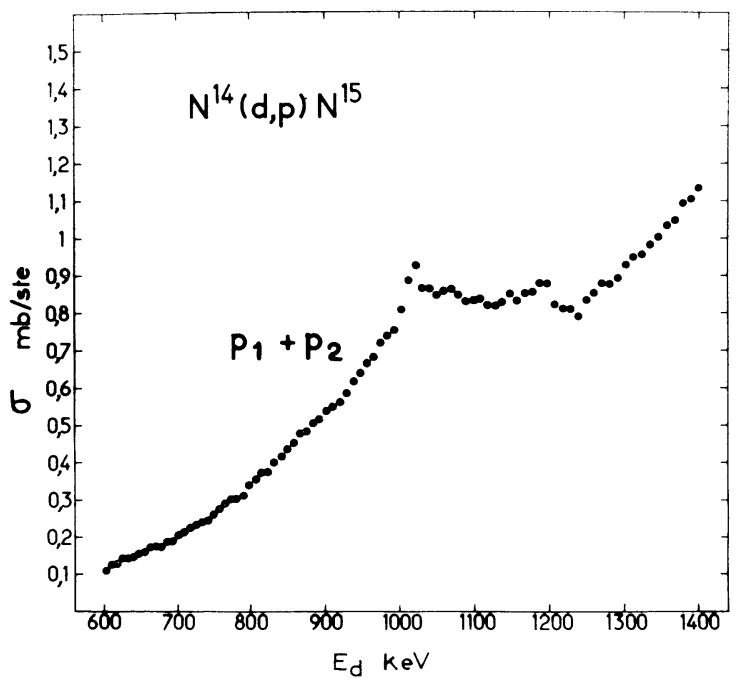

FIG. 4.

Section efficace différentielle à $150^{\circ}$ du doublet $\left(p_{1}+p_{2}\right)$.

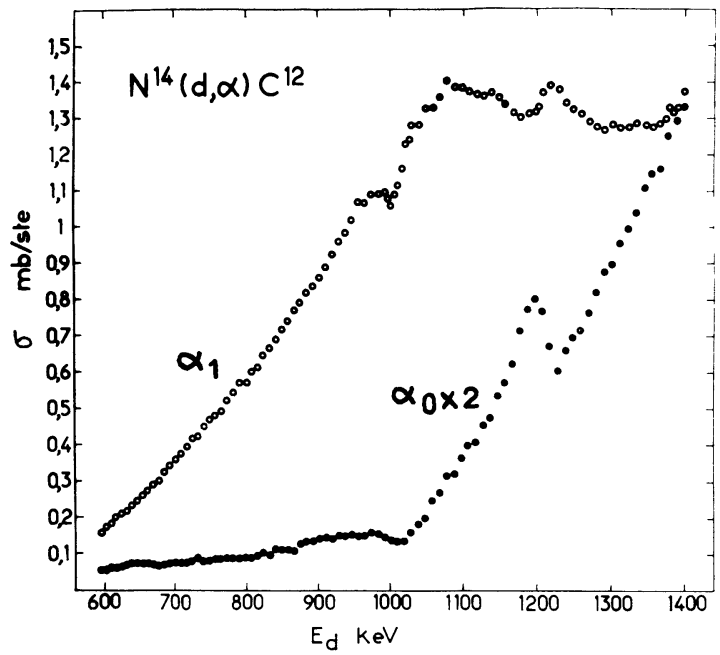

FIG. 5.

Sections efficaces différentielles à $150^{\circ}$ des pics $\alpha_{0}$ et $\alpha_{1}$.

du spectre sur toute la gamme d'énergie explorée. Dans la région de $1100 \mathrm{keV}$, il présente, sinon un plateau, du moins un domaine de variation lente. Une bonne valeur de l'énergie pour doser avec ce pic, dans les cas favorables, est donc $1100 \mathrm{keV}$. Le pic composé $p_{1}+p_{2}$ (fig. 4) présente un bon plateau entre 1000 et $1250 \mathrm{keV}$; son utilisation ne semble cependant pas présenter d'avantages par rapport à celle des rayons $\alpha$. En effet, $\alpha_{1}$ (fig. 5) présente un bon plateau (à quelques petites oscillations près) entre 1100 et $1350 \mathrm{keV}$, avec une section efficace relativement élevée (3 à 4 fois plus faible, il est vrai, que $\left.p_{5}\right)$. Dans le cas général, on utilisera donc le pic $\alpha_{1}$, avec une énergie de bombardement située vers $1300 \mathrm{keV}$. Le pic $\alpha_{0}$ présente un plateau de 600 à $1000 \mathrm{keV}$; la section efficace y est faible et ce n'est que pour les dosages les plus délicats (présence de fluor, par exemple, ou empilements importants, voir plus loin) qu'on fera usage de $\alpha_{0}$ à ces énergies. Il est à noter qu'en passant de 1000 à $1200 \mathrm{keV}$, on multiplie par 6 le rendement de $\alpha_{0}$. Pour favoriser le rendement des couches superficielles de l'échantillon, $\alpha_{0}$ observé à $1200 \mathrm{keV}$ convient donc particulièrement bien.

IV. Conditions pratiques de dosage. - Nous n'examinerons ici que le cas de couches relativement minces (de l'ordre de 1 micron) pour lesquelles une détermination de la teneur globale en azote est requise. La détermination de profils de concentration d'azote sera décrite dans un article à venir.

A. Coughes sur substrats a noyaux lourds. Ces substrats ( $Z$ supérieur à 20 ou 25) ne fournissent aucune réaction. Si la couche elle-même ne contient pas d'autres éléments légers que l'azote (sauf $\mathrm{O}^{\mathbf{1 6}}$ et $\mathrm{C}^{\mathbf{1 2}}$ toujours présents), le dosage peut s'effectuer avec les pics $p_{5}+p_{4}$ ou $\alpha_{1}$, comme cela a été décrit plus 
TABLEAU IV

\begin{tabular}{|c|c|c|c|}
\hline Pia & $\begin{array}{l}\text { ÉNERGIE } \\
\text { OPTIMALE }\end{array}$ & $\begin{array}{c}\text { TAUX } \\
\text { DE } \\
\text { COMPTAGE }(*)\end{array}$ & $\begin{array}{c}\text { SENSIBILITÉ } \\
\text { CALGULÉE }\left(^{* *}\right) \\
\left(\mu \mathrm{g} / \mathrm{cm}^{2}\right)\end{array}$ \\
\hline - & - & - & - \\
\hline$p_{5}+p_{4}$ & $1100 \mathrm{keV}$ & 152 & $5 \times 10^{-4}$ \\
\hline$\alpha_{1}$ & $1300 \mathrm{keV}$ & 34 & $25 \times 10^{-4}$ \\
\hline$\alpha_{0}$ & $1000 \mathrm{keV}$ & 2 & $425 \times 10^{-4}$ \\
\hline$\alpha_{0}$ & $1200 \mathrm{keV}(* * *)$ & 11 & $75 \times 10^{-4}$ \\
\hline
\end{tabular}

\begin{abstract}
(*) Par $\mu \mathrm{g} / \mathrm{cm}^{2}$ d'azote par $\mu \mathrm{C}$ de faisceau, avec un angle solide de 0,12 stéradian.
(**) Épaisseur d'une couche donnant 100 coups en $20 \mathrm{mn}$ avec un courant de $1 \mu \mathrm{A}$ et un angle solide de 0,12 stéradian.

$(* * *)$ Pour des couches minces.
\end{abstract}

haut. On obtiendra des spectres analogues à celui de la figure 1. Les taux de comptage optimaux obtenus dans ces conditions avec un détecteur de $3 \mathrm{~cm}^{2}$ à $50 \mathrm{~mm}$ de la cible $(0,12$ stéradian, une géométrie aisée à réaliser à $150^{\circ}$ ) sont indiqués dans le tableau IV, pour $1 \mu \mathrm{g} / \mathrm{cm}^{2}$ d'azote et pour $1 \mu \mathrm{C}$ (soit par seconde pour $1 \mu \mathrm{A}$, qui est le courant normalement utilisé). En l'absence de bruit de fond et en limitant la durée d'une mesure à $20 \mathrm{mn}$, si on requiert 100 coups dans le pic (précision statistique $10 \%$ ), on peut calculer une sensibilité de détection de l'azote; dans le tableau IV, on indique les quantités minimales d'azote (calculées) qu'on peut ainsi mesurer, en $\mu \mathrm{g} / \mathrm{cm}^{2}$. Le faisceau ayant un diamètre d'environ $1 \mathrm{~mm}$, on peut en déduire, en divisant par 100, la sensibilité absolue en $\mu \mathrm{g}$. Encore faut-il que la cible supporte sans altération $20 \mathrm{mn}$ de bombardement, ce qui est le cas en général, sauf peut-être pour des matériaux d'origine biologique [2]. Notons que la sensibilité calculée pour $p_{5}+p_{4}$ n'est pas atteinte dans la pratique à cause des nombreuses sources de coups parasites aux basses énergies, toujours présentes. Ajoutons que, pour les rayons $\alpha$, on peut aisément utiliser trois détecteurs identiques disposés en couronne, couplés en parallèle. On peut ainsi tripler le taux de comptage sans trop dégrader la forme des pics [8]. La durée d'une mesure est usuellement de l'ordre d'une minute.

Si la couche contient d'autres éléments légers, on est amené à utiliser $\alpha_{1}$, sauf peut-être en présence de $\mathrm{F}^{19}$, $\mathrm{Li}^{7}$ ou $\mathrm{B}^{10}$. Pour dégager $\alpha_{1}$ d'éventuels pics dus à des protons de réactions parasites $(\mathrm{d}, \mathrm{p})$, il faut diminuer l'épaisseur de la zone sensible du détecteur, selon le procédé illustré par la figure 2. Avec une barrière de 120 microns, l'énergie maximale qu'un proton peut laisser dans le détecteur est d'environ $3,5 \mathrm{MeV} ; \alpha_{1}$ est alors toujours dégagé des pics protons $[6,7]$. Un détecteur de $3 \mathrm{~cm}^{2}$, d'une résistivité de $3700 \Omega$. cm, polarisé à environ $10 \mathrm{~V}$, donne des résultats satisfaisants avec un amplificateur à bas bruit classique (Ortec $109 \mathrm{~A}$ par exemple).
B. Coughes sur substrats a noyaux LÉgers. Dans ce cas, le rendement des réactions se produisant sur la cible massive constituée par le substrat peut devenir très grand. Par exemple, lorsqu'on dose de l'azote sur un substrat de quartz, la réaction $\mathrm{O}^{16}(\mathrm{~d}, \mathrm{p}) \mathrm{O}^{17^{*}}$ sur l'oxygène peut aisément fournir $10^{5}$ coups/s. Le fait que l'énergie des particules parasites est nettement inférieure à celle de $\alpha_{0}$ ou $\alpha_{1}$ n'est plus alors une protection suffisante, les phénomènes d'empilement dans la chaîne électronique pouvant complètement masquer les pics étudiés. Il n'est alors pas question d'utiliser $p_{5}+p_{4}$, et même $\alpha_{1}$ peut devenir difficile à observer. On peut cependant, dans la plupart des cas, utiliser $\alpha_{0}$, à condition de prendre quelques précautions. Une solution peut être d'opérer à plus faible énergie : la section efficace des réactions parasites peut décroître très vite. On peut aussi réduire le courant de faisceau au-dessous du $\mu \mathrm{A}$, mais cette possibilité est limitée. Le vrai remède consiste à augmenter la vitesse de l'électronique utilisée. Comme le problème du bruit électronique ne se pose pas pour $\alpha_{0}$ (pic bien isolé et déjà élargi par l'action du mylar), on peut utiliser des constantes de temps de différentiation faibles, 0,1 à $0,25 \mu$ s typiquement. Un amplificateur à seuil élimine alors les impulsions parasites, avant l'analyse. L'utilisation d'un système de réjection d'empilement peut s'imposer. Enfin, dans les cas les plus difficiles, les techniques d'amplification ultra-rapides, mises au point pour des expériences analogues [9], peuvent toujours résoudre le problème. Notons qu'avec un équipement classique, le dosage de l'azote sur du quartz ou du silicium a été aisé.

V. Applications. - A. Pulvérisation cathodique RÉAGTIVE. - Cette méthode de dosage a été appliquée à l'étude des nitrures de silicium et de tantale, formés par pulvérisation cathodique réactive. Les installations utilisées comportaient une cathode massive faite du matériau à pulvériser, et la décharge s'effectuait dans un mélange d'azote et d'argon. 
La composition des gaz introduits dans l'enceinte et la propreté de celle-ci ont une influence déterminante sur la nature du dépôt obtenu.

C'est ainsi que du silicium, pulvérisé dans un mélange d'argon et d'azote renfermant des traces d'oxygène, se combine uniquement avec ce dernier pour donner une couche d'oxyde pur, comme l'ont montré nos expériences.

a) Influence de la pression d'azote sur la composition des dépôts. - Lorsqu'on examine la variation avec la pression d'azote des propriétés physiques des dépôts, telles que la résistivité, on observe un palier dans un domaine de pression souvent assez étendu [10]. On peut se demander si un tel palier apparaît également dans la relation liant la teneur en azote du dépôt à la pression de pulvérisation et, dans l'affirmative, quelle est la composition du dépôt dans cette région. La teneur en azote de couches de nitrure de silicium et de tantale a été étudiée dans une vaste gamme de pressions partielles d'azote. Les résultats numériques dépendent de l'installation utilisée, mais dans tous les cas des paliers ou, du moins, des domaines à faible variation ont été mis en évidence. Ges paliers correspondent bien à ceux observés pour les propriétés physiques. Il est donc souhaitable de travailler dans le voisinage de ces pressions, de façon à obtenir une bonne reproductibilité des couches formées. Les résultats qui suivent sont typiques et illustrent bien ces constatations.

La figure 6 correspond à des couches de nitrure de silicium déposées sur des plaquettes de tantale. Chaque pulvérisation a duré $10 \mathrm{mn}$ et l'épaisseur des films, mesurée par interférométrie, est de l'ordre de $2200 \AA$. Les pressions indiquées sont des pressions partielles d'azote, mesurées avant et après la décharge, en

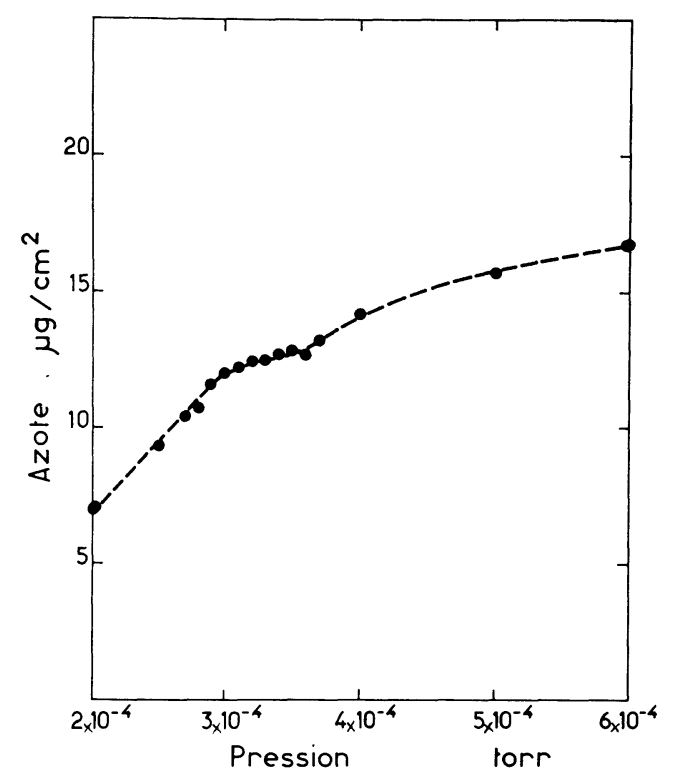

FIG. 6. - Teneur en azote de couches de nitrure de silicium, déposées par pulvérisation cathodique réactive, en fonction de la pression partielle d'azote. l'absence d'argon, avec une variation d'une mesure à l'autre inférieure à $0,1 \times 10^{-4}$ torr. La pression partielle d'argon était de $10^{-2}$ torr. Le palier, qui n'est ici qu'une région de moindre pente, s'étend de 2,9 $\times 10^{-4}$ à $3,5 \times 10^{-4}$ torr. Les mesures semblent indiquer que la composition près du palier correspondrait à $\mathrm{Si}_{2} \mathrm{~N}$; ce résultat doit être confirmé par une mesure plus précise de la masse des dépôts, la mesure d'épaisseur ne fournissant pas une façon sûre d'estimer la teneur en silicium.

La figure 7 concerne des couches de nitrure de tantale déposées sur des plaquettes de silice, également pendant $10 \mathrm{mn}$, mais avec une installation différente.

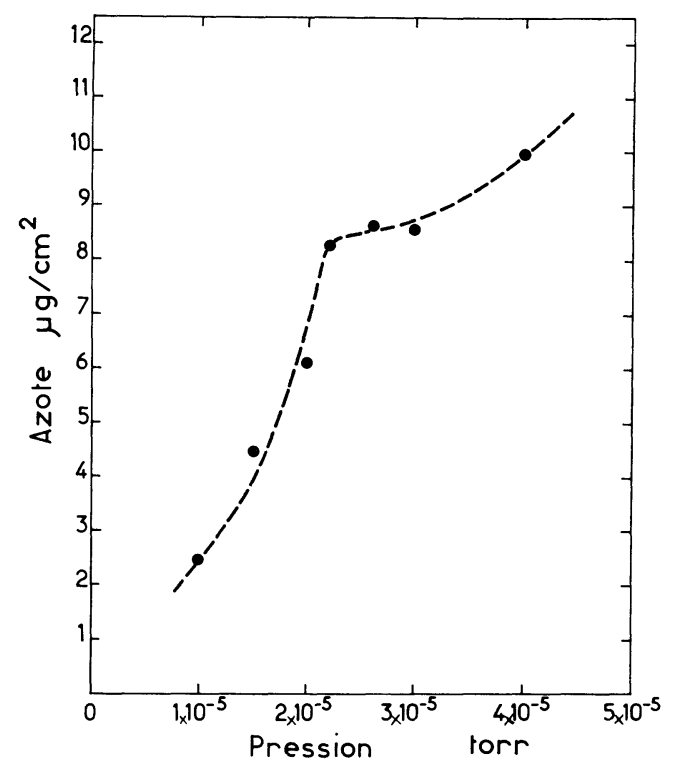

Fig. 7. - Teneur en azote de couches de nitrure de tantale, déposées par pulvérisation cathodique réactive, en fonction de la pression partielle d'azote.

Nous indiquons les pressions partielles d'azote, la pression d'argon étant ici de $2 \times 10^{-2}$ torr. L'épaisseur des couches, en poids par $\mathrm{cm}^{2}$, a pu être déterminée ici avec précision par pesée, vu leur densité élevée; elle était de $0,23 \mathrm{mg} / \mathrm{cm}^{2}$ et correspondait à une épaisseur géométrique d'environ $1400 \AA ̊$. La composition de ces couches sera discutée plus loin.

Dans ces deux cas, nous avons vérifié la constance de la vitesse de pulvérisation de la cathode, quelle que soit la pression partielle d'azote. Dans le cas du tantale, en particulier, des couches déposées à temps constant et à pression variable ont le même poids à $8 \%$ près, et c'est donc bien la teneur relative en azote qui est mise en évidence par la méthode nucléaire.

b) Influence de la durée de la pulvérisation. - Après avoir déterminé quelle est la meilleure pression d'azote à utiliser pour chaque sorte de pulvérisation, nous avons vérifié que la composition de la couche ne variait pas au cours du dépôt. Nous présentons ici les résultats 
relatifs au tantale. L'installation utilisée est la même que celle qui a permis d'établir la courbe de la figure 7 . A une pression partielle d'azote de $2,3 \times 10^{-5}$ torr, 5 couches de nitrure de tantale ont été déposées sur des plaquettes de silice, pendant des durées respectives de $5,10,30$ et $60 \mathrm{mn}$. Ce dernier échantillon a été fait en double, avec une variation de poids de $1,6 \%$ entre les deux couches. La figure 8 montre la variation de la

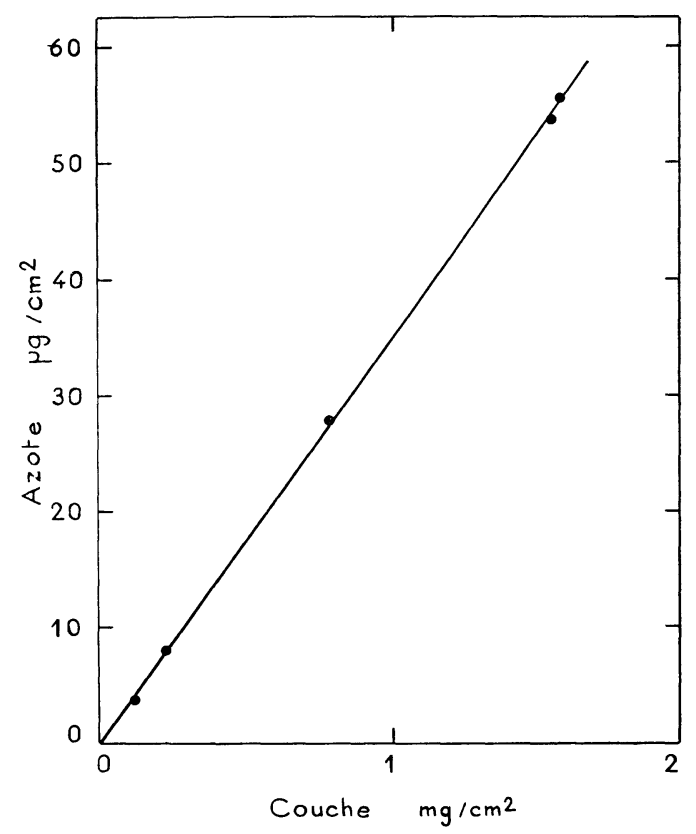

FIG. 8. - Teneur en azote de couches de nitrure de tantale, déposées par pulvérisation cathodique réactive, en fonction du poids de ces couches, à pression partielle d'azote constante.

teneur en azote en fonction du poids des dépôts. On en observe la très bonne linéarité, ce qui permet de conclure que les couches sont homogènes suivant leur épaisseur.

c) Composition des nitrures de tantale. Étalons. - La méthode nucléaire de microanalyse permettant d'effectuer des mesures relatives, il est nécessaire, comme on l'a vu, de disposer d'un étalon renfermant une masse d'azote connue. Celle-ci ne peut être mesurée, en valeur absolue, que par des procédés chimiques.

Pour obtenir un tel étalon, nous avons réalisé une série de couches de nitrure de tantale, de poids connus, voisins de $0,3 \mathrm{mg} / \mathrm{cm}^{2}$, déposées sur des lames d'environ $20 \mathrm{~cm}^{2}$. La pression partielle d'azote pour chaque dépôt était différente, et comprise entre $1,1 \times 10^{-5}$ et $1,8 \times 10^{-5}$ torr, valeurs encadrant le palier de concentration pour l'installation utilisée. Ces échantillons, groupés par paires pour augmenter la masse d'azote, ont été dosés chimiquement par les soins du Bureau Véritas. En prenant la moyenne des résultats, nous avons pu attribuer à ce nitrure la formule $T a_{2,5} \mathrm{~N}$.
Il s'agit donc d'une variété sous-stœchiométrique, en azote, de $\mathrm{Ta}_{2} \mathrm{~N}$.

Avant leur destruction par l'analyse, les échantillons avaient été comparés, par la méthode nucléaire, à la couche mince de nitrure de tantale qui nous a permis de mesurer les sections efficaces. Nous avons donc pu calculer la teneur en azote de celle-ci, et la prendre ensuite comme référence. Pour améliorer la précision de cet étalon, nous comptons effectuer de nouvelles expériences, dont le dosage nucléaire du tantale par diffusion élastique et comparaison à une mince couche de tantale pur. Actuellement, nous estimons que la précision de notre étalon est de $\pm 10 \%$; il contient $7,1 \mu \mathrm{g} / \mathrm{cm}^{2}$ d'azote.

B. Nitruration thermique. - Nous présentons ici les premiers résultats d'une étude destinée à élucider le mécanisme de nitruration de certains métaux traités thermiquement dans l'air ou dans l'ammoniac.

A la faveur de l'étude de l'oxydation du zirconium, nous avons recherché l'azote dans des échantillons de ce métal, recuits dans différentes conditions de température et de pression. En présence d'oxygène et d'azote, à la pression atmosphérique, le zirconium se combine de préférence avec l'oxygène et sa nitruration est très faible. C'est le cas d'échantillons recuits à $788^{\circ} \mathrm{C}$ dans l'air sous pression atmosphérique, de $5 \mathrm{mn}$ à $14 \mathrm{~h}$, dont l'oxyde contenait moins de $2 \times 10^{-4}$ d'azote en rapport de poids. Si le traitement chimique a lieu à plus faible pression $\left(100 \mathrm{~g} / \mathrm{cm}^{2}\right)$, dans une atmosphère d'oxygène contenant $5 \%$ d'azote, il semble, au contraire, que la nitruration soit plus forte. Ce phénomène dépendrait donc de la pression partielle d'azote; une étude détaillée est en cours.

Des mesures ont été également effectuées sur des échantillons traités à $800^{\circ} \mathrm{G}$ dans l'ammoniac pendant $10 \mathrm{mn}$. Le but était de rechercher quel métal et quel traitement conviendraient le mieux à la réalisation de couches minces de nitrures métalliques utilisables comme étalons. Nous avons ainsi observé que le tantale, le titane et le zirconium se nitruraient fortement dans ces conditions. Sur le silicium, un film mince de quelques couches moléculaires se forme, mais il ne semble pas qu'il y ait de nitruration en profondeur. Ce résultat est à comparer à ceux obtenus par la pulvérisation cathodique, qui produit des dépôts appréciables de nitrure de silicium. Enfin, le nickel est pratiquement insensible à l'action de l'ammoniac. Il peut donc être utilisé comme support d'une couche métallique avide d'azote, permettant de réaliser des couches minces de nitrure saturées et d'obtenir, par exemple, des couches étalons plus riches que celles fournies par la pulvérisation cathodique.

Mentionnons encore une application de ces techniques à l'étude de l'oxydation anodique du tantale dans l'acide nitrique décrite dans [11]. Dans de l'acide concentré, de l'azote est massivement incorporé; cela peut fournir un autre moyen de construction de cibles étalons. 
Conclusion. - La méthode de microanalyse par l'observation directe des réactions nucléaires s'applique remarquablement bien au cas de l'azote, en raison des fortes sections efficaces conduisant à une bonne sensibilité et de l'existence d'une réaction engendrant un pic d'énergie élevée, peu susceptible d'être masqué par des réactions parasites. Les effets de ces dernières peuvent presque toujours être annulés par un choix convenable des conditions expérimentales. L'existence de domaines d'énergie où les sections efficaces des réactions varient peu permet de doser dans de bonnes conditions des couches relativement épaisses. L'analyse est rapide et non destructive et trouve des applications nombreuses en physique des solides, en métallurgie et même éventuellement en biologie. Elle présente des avantages marqués par rapport aux techniques d'évaporation flash par un éclair lumineux, suivie d'une analyse par chromatographie cryogénique des gaz libérés [12]; en particulier elle peut remplacer de telles techniques pour l'étude des couches de nitrure déposées sur des semiconducteurs, de plus en plus utilisée dans l'industrie électronique moderne. En effet, les méthodes de flash ne semblent pas donner une précision meilleure que $10 \%$ et demandent environ $1 \mathrm{~h}$ par dosage.

Gette méthode est complémentaire des techniques d'analyse par activation. Gelles-ci [13 à 17] requièrent des énergies de bombardement élevées si on veut une bonne sensibilité et exigent donc la mise en œuvre de moyens beaucoup plus importants. En revanche, cette énergie élevée elle-même est un avantage si on veut mesurer de très faibles concentrations d'azote dans la masse des échantillons. C'est ainsi que des couches de plusieurs millimètres d'un échantillon peuvent être explorées avec des protons énergiques et que l'irradiation par des photons d'une vingtaine de $\mathrm{MeV}$ peut intéresser tout le volume de gros échantillons. Des traces d'azote très inférieures à 1 p.p.m. peuvent donc être mesurées par activation $[14,16]$. Il est clair que la méthode décrite ici s'applique avec avantage au cas fort différent des couches minces près de la surface des échantillons, mais ce n'est que d'une utilité réduite pour la mesure de faibles concentrations en volume.

Sans revenir longuement sur le cas des sondes ionique et électronique, déjà discuté $[2,5]$ pour l'oxygène, similaire à l'azote de ce point de vue, on peut mentionner que ces méthodes peuvent également se révéler complémentaires de la méthode nucléaire. Par leur haute résolution latérale, que la technique décrite ici ne peut pas avoir, ces méthodes peuvent fournir des indications irremplaçables dans les études de structure. La sonde électronique, dont l'emploi ne peut être étendu au traçage par l'isotope $\mathrm{N}^{15}$, présente cependant pour l'azote une sensibilité bien plus faible, surtout sur substrat de $Z$ élevé. La sonde ionique [18] a un rendement qui dépend fortement de la matrice contenant l'élément à doser; les résultats ne peuvent pas être rendus quantitatifs par un étalonnage universel. C'est ainsi que la comparaison directe de couches de composition inconnue et variable (telles que les nitrures formés à diverses pressions d'azote étudiées dans cet article) serait difficile avec cet instrument. Un dosage global nécessiterait l'érosion de toute l'épaisseur de la couche avec intégration du signal, ce qui peut poser des problèmes délicats. Cependant, dans bien des cas, les techniques d'analyse par sonde peuvent se révéler avantageuses; par ailleurs, elles présentent une accessibilité pratique plus grande. Les comptes rendus d'une récente table ronde consacrée aux méthodes d'analyse [19] permettent de situer ces diverses techniques et d'évaluer la meilleure façon d'en tirer parti.

Remerciements. - MM. Croset et Kleefstra, du Centre de Recherches Physico-chimiques de la C.S.F. à Puteaux, nous ont apporté une aide inappréciable pour les expériences portant sur la pulvérisation cathodique réactive. Nous remercions également $\mathrm{M}$. Laurent, de la Faculté des Sciences d'Orsay, pour son concours aux expériences de nitruration dans l'ammoniac.

\section{BIBLIOGRAPHIE}

[1] AMSel (G.), Rapport L.A.L. 1053, mai 1963, Orsay.

[2] Amser (G.) et Samuer, (D.), Anal. Chem., 1967, 39, 1689.

[3] NADAI (J. P.), Thèse, Orsay, 1967.

[4] Amsei, (G.), BÉRANGER (G.), DE Geilas (B.) et Lacombe (P.), J. Appl. Phys., 1968, 39, 2246.

[5] Amsei, (G.), David (D.), BÉRANGer (G.) et BoISOT (P.), Rev. Phys. Appl., 1968, 3, 373.

[6] Amsel (G.), Baruch (P.) et Smulkowski (O.), Nucl. Instr. Meth., 1960, 8, 92.

[7] AMSel (G.), Baruch (P.) et Smulkowski (O.), I.R.E. Trans. Nucl. Sci., NS 8, 1, 21, janvier 1961.

[8] AMser (G.) et NADAI (J. P.), article général à paraître.

[9] Amser, (G.), Bosshard (R.) et ZAJDE (C.), I.E.E.E. Trans. Nucl. Sci., NS 14, 1, 1, février 1967.

[10] KLEEFSTRA (M.), Le vide, no spécial A.V.I.S.E.M., octobre 1966, 95.
[11] Amser (G.), Cherki (C.), Feuillade (G.) et NADAI (J. P.), à paraître dans J. Phys. Chem. Solids.

[12] Guldner (W. G.) et BRown (R.), Measurement techniques for thin films, Electrochemical Society, New York, 1967, 82 (Conférence de Philadelphie, octobre 1966).

[13] AlberT (Ph.), 5e Colloque de Métallurgie de Saclay (juin 1961), P.U.F., p. 37.

[14] Albert (Ph.), Engelmann (Ch.), May (S.) et Petit (S.), C. R. Acad. Sci., 1962, 254, 119.

[15] Eingelmann (Ch.), Bull. Soc. Chim. France, 1967, 7, 2316.

[16] Cabane (G.) et Engelmann (Ch.), Rev. Phys. Appl., 1958, 3, 365 .

[17] Markowitz (S.) et Mahony (S.), Anal. Chem., 1962, 34, 329

[18] Stodzian (G.), Rev. Phys. Appl., 1968, 3, 360.

[19] Numéro spécial Rev. Phys. Appl., 1968, 3, 307 à 386. 